\title{
DISCOVERY OF CEPHEIDS IN M100 AND M101
}

\author{
JEREMY MOULD \\ Mount Stromlo and Siding Spring Observatories \\ Institute of Advanced Studies \\ Australian National University \\ WENDY FREEDMAN \\ Observatories of Carnegie Institution of Washington
}

LAURA FERRARESE

Johns Hopkins University and Space Telescope Science Institute

AND

DAN KELSON

University of California, Santa Cruz

\section{Introduction}

The Hubble Space Telescope key project to determine the extragalactic distance scale aims to measure $\mathrm{H}_{0}$ to $10 \%$. To achieve this goal will require Cepheid distance measurements for some 20 galaxies within a redshift of approximately $10^{3} \mathrm{~km} / \mathrm{s}$. These galaxies in turn will calibrate five secondary distance indicators which will extend the volume over which the expansion rate has been measured to some $10^{6} \mathrm{Mpc}^{3}$.

\section{M101}

We observed the Cook field in M101 with WF/PC in April 1993. Kelson et al (1995) have identified 29 Cepheids in the field. Light curves of six of these stars are shown in Figure 1.

\section{3. $\mathrm{M} 100$}

M100 is a member of the Virgo cluster. The galaxy was observed as an Early Release Observation during the first days of WFPC2 (Freedman et al 1994a, Trauger et al 1994) and reobserved intensively in April 1994. Ferrarese 

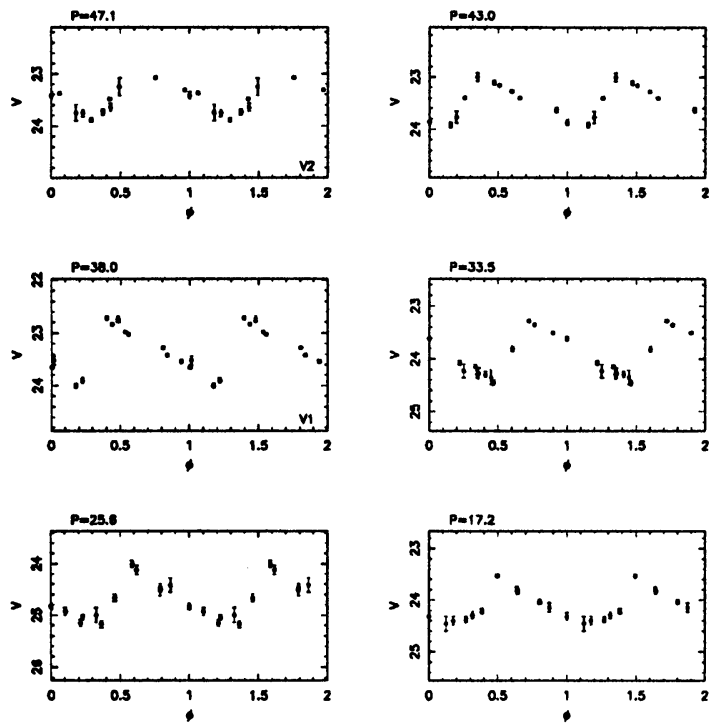

Figure 1. M101 Cepheids showing periods in days.

et al (1995) and Hill et al (1995) have described the identification and measurement of Cepheids in this galaxy. Light curves of two of these stars are shown in Figure 2. The Period-Luminosity relation for M100 Cepheids is reported by Freedman et al (1994b and this volume).
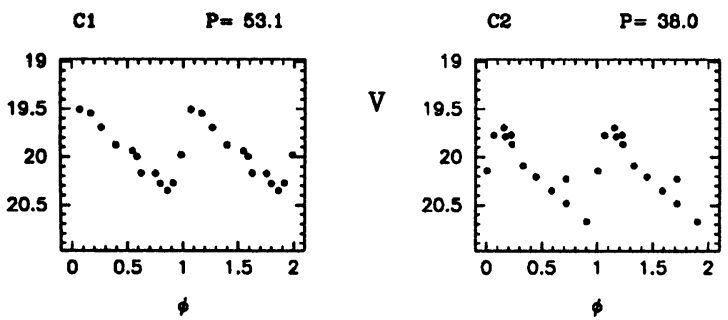

Figure 2. Light curves of 2 M100 Cepheids showing instrumental magnitudes.

We are grateful to all our colleagues on the $\mathrm{H}_{0}$ Key Project team for their contributions. The project is supported by STScI Grant 2227 from NASA.

\section{References}

Ferrarese, L. et al. 1995, ApJ, in press

Freedman W. et al. 1994a, ApJ Letters in press

Freedman W. et al. 1994b, Nature, Oct. 27, 1994

Hill, R. et al. 1995, ApJ, in press

Kelson, D. et al. 1995, ApJ, in press

Trauger, J. et al. 1994, ApJ Letters, in press 